$\$ \equiv$

\title{
Endodontic flare ups: A proposal for etiopathogenesis and psychological management
}

\author{
Pushappreet Kaur \\ Dental officer, Guru Nanak Dev Dental Hospital, Sultanpur Lodhi, Kapurthala, Punjab- 144626 \\ E-mail: kaurpushp@gmail.com
}

\begin{abstract}
Flare ups are undesirable yet unavoidable, true endodontic emergencies, presenting with severe pain and/or swelling of the facial soft tissues and the oral mucosa in the area of endodontically treated tooth, in association with strong expression of clinical symptoms (tooth pain when biting, chewing or by itself), occuring within a few hours or a few days of instrumentation. Prevention and management of such situations necessitates standardized execution of all the steps comprising root canal therapy alongwith comprehensive knowledge of its possible etiological factors, pathogenesis, pharmacological and non-pharmacological treatment methods. This article presents a proposal for etiopathogenesis and psychological management besides accounting a brief description of its definition, epidemiology, treatment steps and prevention.
\end{abstract}

Keywords: Etiopathogenesis; Flare Up; Psychological Management; Root Canal Therapy.

\section{Introduction}

The preliminary goal of endodontic treatment is biomechanical preparation of the root canal which is achieved by its cleaning, shaping and disinfection, thereby reversing the disease process along with elimination of associated signs and symptoms and followed by its hermetical obturation without additional periradicular injury or discomfort to the patient, thus providing optimal conditions for the periradicular tissues to heal [Senia 2000, Sathhorn et al. 2007, Udoye et al. 2010]. Mild discomfort or pain between appointments and/or postoperatively is a common occurrence but acute exacerbation of pain and swelling is unusual and requires immediate management. Such endodontic emergency is called "Flare up" and it is annoying to the patient, particularly if the tooth was asymptomatic prior to treatment, forcing him/her to call the dentist office to arrange an unscheduled and emergency appointment, thus distressing the dentist and disrupting a busy practice. Fortunately, the occurrence of a flare-up does not lower the prognosis of endodontic treatment.

\section{Definition}

A flare-up can be defined as pain and/or swelling of the facial soft tissues and the oral mucosa in the area of the endodontically treated tooth, that occur within a few hours or a few days following the root canal treatment, when clinical symptoms (tooth pain when biting, chewing or by itself) are strongly expressed and the patient visits a health care institution sooner than scheduled [Pasqualini et al. 2012]. Even after following standard procedural protocols, varying degrees of residual pain can be expected postoperatively and should be accepted and anticipated by the patients. In some instances, flare-ups may occur following an access opening without root canal instrumentation. It is a poor indicator of pathosis and unreliable predictor of long-term success [Zuckerman et al. 2007].

\subsection{What is the difference between postoperative pain and flare up?}

Postoperative pain and flare up are two terms used commonly to describe inter-appointment pain, but there is the difference between the two. Postoperative pain is defined as pain of any degree that occurs after initiation of RCT, whereas flare-up has been defined as the onset or continuation of pain and/or swelling after endodontic treatment. Flare-up is subset of postoperative pain [Sathorn 2007]. Patients might consider postoperative pain and flare-up as a benchmark against which the clinician's skills are measured. It might undermine patients' confidence in their dentists or patient satisfaction with the treatment. Hence, prevention and management of post endodontic pain is considered an integral part of endodontic treatment. Informing patients about expected post endodontic pain (PEP) and prescribing medications to manage it can increase patient confidence in their dentists, increase patients' pain threshold, and improve their attitude toward future dental treatment [Van Wijk et al. 2006]. PEP (not limited to flare up) is very frequent after endodontic treatment, and more than $50 \%$ of those who feel any PEP experienced severe pain [Levin et al. 2006].

According to litreature, the flare-up rate varied from $1,4 \%$ to $16 \%$ and up to 50\% [Oliveira Alves 2010]. The reason behind this wide variation in frequency was the difference in study types (prospective and retrospective) and methodology, time of tooth pulp, apical periodontitis diagnosis, the moment the pain was recorded and the clinical experience with the dentist and his/her practical skills. Females, patients on steroids for systemic diseases, cases which present preoperative pain and tenderness and lateral teeth (molars) show higher frequency of flare ups [Glennon et al. 2004]. 


\section{Etiopathogenesis}

Etiopathogenesis of flare up can be explained as a repercussion of interaction between a variety of host, microbial and procedural factors. This article attempts at a proposal for etiopathogenesis in flow chart form as shown below in Fig.1 and Fig.2.

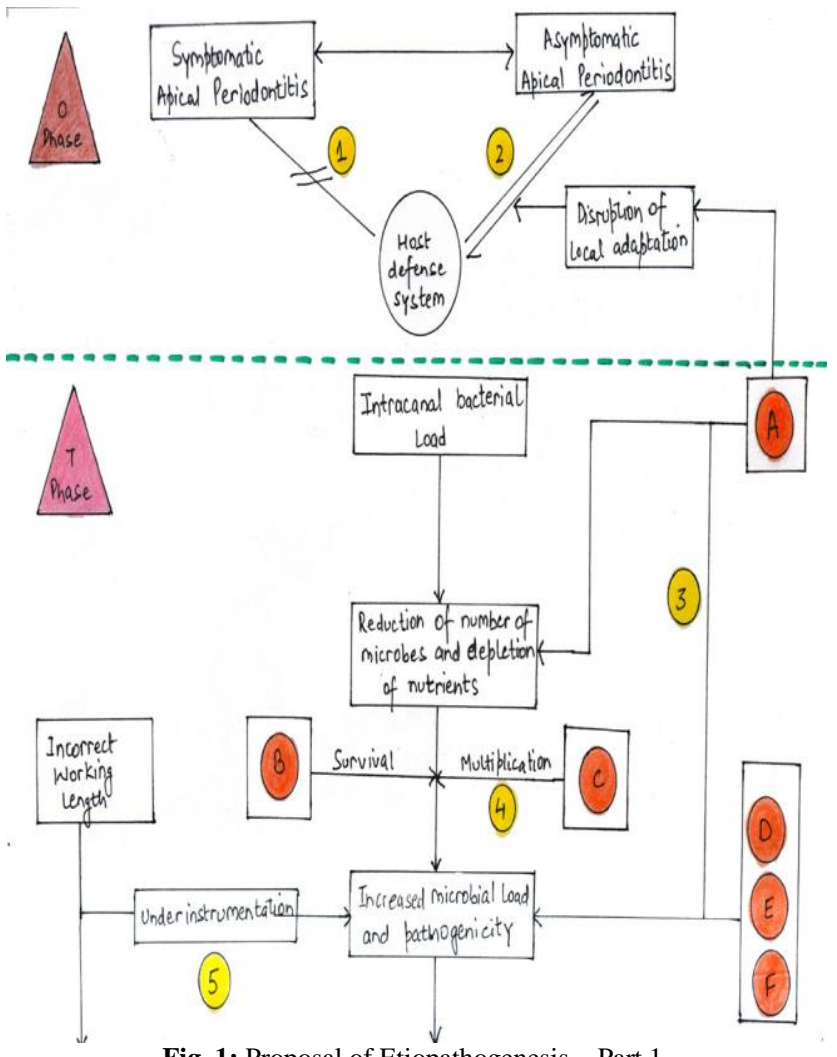

Fig. 1: Proposal of Etiopathogenesis - Part 1.

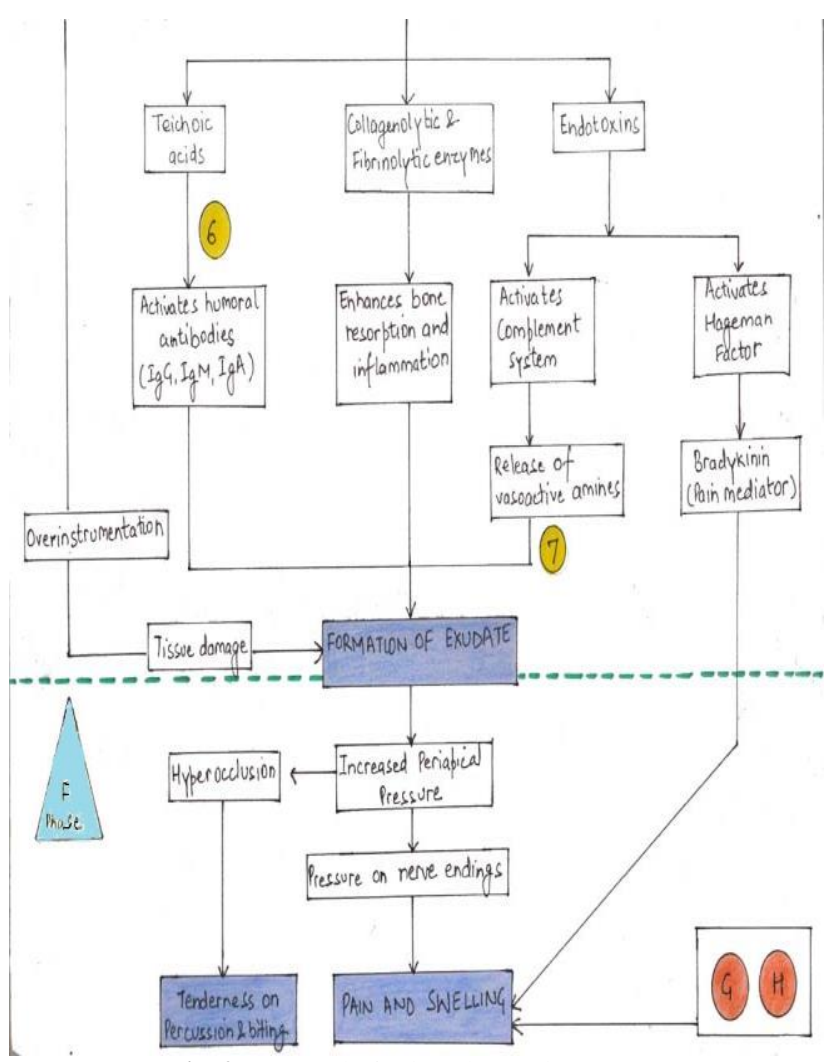

Fig. 2: Proposal of Etiopathogensis - Part 2.

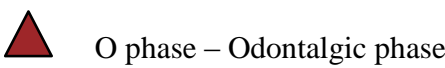

$\triangle$

T phase - Treatment phase

F phase - Flare up phase

1) Presentation of preoperative pain and swelling in symptomatic apical periodontitis occurs on account of interaction between periapical microflora and host defense.

2) $\bigcirc$ Absence of preoperative pain and swelling in case of asymptomatic apical periodontitis can be fairly explained by phenomena called "Local adaptation," which refers to development of balance between microbial aggression and host defense in a chronically inflamed tissue. Instrumentation causes extrusion of infective microbes, which act as new irritants and disturb this balance thereby initiating a host response [Selye 1953].

3) $\bigcirc$ Mechanical preparation errors: All methods of instrumentation have been found to cause concomitant apical extrusion to variable extent, which may be exaggerated by use of compressed air and injudicious pressure while irrigating. Additionally, inadequate debridement, irrigation and medicaments also result in retention of infective bacteria which in presence of some strains might exhibit increased virulence.

4) $\bigcirc \quad$ Multiplication of residual microflora is facilitated by the presence of blood and serum (host factors).

5) $O$ Under instrumentation (short working length) results in incomplete elimination of bacterial agents.

6) $\bigcirc$ Teichoic acids are found in the cell wall and plasma membrane of gram positive bacteria. In addition to them, some root canal medicaments and sealants also might provoke an immunologic response.

7) $O$ Capillary permeability is increased by the action of vasoactive amines like histamine and serotonin.

a) Mechanical preparation: Causes reduction in the intra canal bacterial overload.

b) Bacterial survival methods [Fabricius et al. 1982]: Ntr gene system; Arc system; Catabolic repressor system; Utilization of organic and inorganic phosphate; Biofilm formation; Resistance of ingestion by PMNs; Oxygen tension dependent adaptation ability.

c) Multiplication methods: Numerous studies involving culture of intracanal microflora are available in literature, but reproduction methods are scarcely documented. However, bacterial methods of reproduction are generally divided into:

Vegetative - budding, binary fission and fragmentation

Asexual - spore formation

Sexual - transduction, conjugation and transformation

d) Iatrogenic introduction of microbes: Use of contaminated irrigants, medicaments, paper points and non sterilised endodontic instruments as well as working without rubber dam.

e) $\bigcirc$ Migration of dormant bacteria: Bacteria residing in anatomical areas of the root canal such as isthmuses, apical ramifications, irregularities, deltas and dentinal tubules may remain unaffected by disinfection procedures [Siqueira et al. 1996] and survive by deriving nutrients from tissue remnants, although some might be killed or prevented from gaining access to the periradicular tissues after the canals are obturated. However, if the obturation seal is inadequate (under extended), it may allow seepage of tissue fluids providing a substrate for bacterial growth, which, then gain access to the periradicular area and cause periapical inflammation [Siqueira 2001].

f) $\mathrm{O}$ Entry of microbes via oral cavity: Ignored carious tissue; Old non hermetic filling; Crack in temporary restoration; Fracture of tooth structure.

g) $\bigcirc$ Trauma afflicted by overfilling: Overextension of gutta percha points and paper points; Overfilling of permanent restoration; High point in the altered occlusion due to considerable removal of tooth structure during access preparation.

h) Hypersensitive response: It is an individualistic response and may occur with intracanal medicaments, root canal 
sealers and irrigants. Periapical extrusion of irrigants might initiate similar response.

\section{Management}

Flare ups constitute an undesirable aspect of endodontic treatment and are true emergencies, which require immediate attention. Successful management of such emergencies is an important part of dentistry and somehow helps re-establishment of patient's trust in his/her dentist, becoming critical for one's private practice. There are various aspects regarding management of these situations which should be managed skillfully in a stepwise manner.

\subsection{Psychological management}

Fear and anxiety are closely associated with magnification of pain perception, rationalizing the importance of psychological counseling along with treatment of flare up to remove any doubts and fear in patient's mind, which are affirmed by regression of pain and swelling comprising flare up. Psychological conditioning or preparation is an under the practiced and underestimated approach when it comes to be endodontic flare up management. This approach, however, if implemented tactfully can very well supplement pharmacological and surgical management.

\subsubsection{Importance of psychological counseling}

Flare up can be safely described as an acutely painful condition which stirs up a variety of negative emotions in the patient, most commonly fear, anxiety, anger, doubt and loss of control, which eventually cohere and shape his/her state of mind. A mind brimming with this brew of emotions expectedly reacts on them, making random speculations like extraction of involved tooth or failure of treatment, which subsequently amplify a current mental state. Resultantly, when the patient arrives at the dental office, he/she is quite scared, anxious, feeling out of control and is uncertain about the upcoming events. It is important that the surgeon should anticipate such reactions and plan the treatment strategies accordingly. Psychological counseling is an approach which helps the surgeon gradually orient patient's state of mind in a positive direction from their present state of fear and anxiety. Since fear and anxieties are directly related to the perception of pain, one can expect successful management of pain if there is reduction in the level of anxiety and fear. There are few methods, which can be integrated before, during and after clinical procedures:

\subsubsection{Addressing the problem}

The foremost step of psychological management involves addressing the problem. It is important that the patient should vent out all negative emotions and express his/her feelings. It also helps the dental practitioner to get an insight of patient's psychological state and their level of fear and anxiety. The conversation should be started by the dentist and then subtly be oriented towards history taking, asking questions about pain. Acknowledgements of the problem by the dentist make the patient feel engaged and shown a sense of concern on the part of dentist who helps rebuild patient's trust, confidence as well as generates a sense of security. A possible description of this reason is that during such situations, the patient is doubtful regarding capabilities of the dentist and is not expecting any kind of acknowledgement or plausible explanation from his part. So, when this happens, patient is taken by surprise, feels encouraged and redeems vague balance and composure.

\subsubsection{Physical gestures}

Body language of the dentist also projects care and concern in addition to words. Touching the patient at the level of shoulders, maintaining direct eye contact along with a soft tone of voice all together build a calm and relaxing atmosphere which helps lower down patient's anxiety thereby leveling the heightened perception of pain.

\subsubsection{Providing information}

Anxiety and fear capitalizing mind of patient in pain, originate from the uncertainity about the upcoming events and its consequences. Unexplained speculations and anticipations generate insecurity and make them feel out of control. The surgeon should explain flare-up in a simple and understandable language using layman terms. Information can also be conveyed using informatory videos and literature as well case reports depicting successful management of such situations. The surgeon must explain that a flare up is less common but expected complication of endodontic treatment, which is totally manageable and does not affects the outcome of treatment. Aside from this, providing sensory information about the procedure to be performed also decreases anxiety and fear in patient. Patient should be informed what to expect during that procedure.

\subsubsection{Psychological evaluation of people accompanying:}

Psychological status of the person accompanying the patient forms an important external factor influencing patient's pain and anxiety level. Raised level of anxiety and fear in the person accompanying develops emotional distress and might hinder the development of confidence, security and calmness in the patient. Therefore, it is preferable to ask for any accompanying visitor to wait in the reception area rather than in the operatory.

\subsubsection{Choice of words}

Words should be chosen very carefully while communicating with patients, especially those in acute pain because they play a vital part in establishment of a secure and calm atmosphere, orienting the patient in a positive direction. An illustration has been given using a simple example which presents two conversations under similar circumstances but with a different choice of words.

"Flare ups are very well known complications of endodontic treatment. But, there is nothing to worry, we will find the cause of problem and after that you will be fine".

"Let me first assure you that there is nothing you need to worry about. Nothing is wrong with your treatment. Consider this a small speed bump on your road to recovery and it does not affect the final outcome of your treatment at all. I will try my best to explain this event, which is medically described as flare up. If you still have any questions, please feel free to ask. After that I will perform the necessary clinical examination which is very much a routine work, and you are already familiar with. Hopefully after this session, you will be back on track in a day or two."

The second conversation is definitely more comforting for the patient and works well towards lowering the level of anxiety and pain compared to first one.

\subsubsection{Eliminating any other pain triggering external stimuli}

Make sure that the patient is in complete isolation and is not a witness to any procedure involving physical display of reluctance and oppression been performed in vicinity. In the context of our psychological development, screaming, shouting and crying are automatically processed and associated with threatening stimuli thereby further elevating previously raised level of anxiety. These events finally lead to intensification of pain.

\subsection{Ruling out other causes}

It is important to rule out other possible causes/conditions which might present similarly to flare up in the form of pain and/or swelling.

\subsubsection{Pulpal and periodontal conditions}




\subsubsection{Acute pulpitis}

This condition if present in any tooth on the involved side can produce severe pain on the entire side which may radiate to temple, ear or neck, closely resembling an endodontic flare up (with presentation of searing pain without swelling). The chances of missing such diagnosis are extremely slight although it may happen if the tooth in question lied adjacent to the tooth being endodontically treated, was previously filled and mildly sensitive and somehow developed the condition coincidently in the inter appointment period. Fortunately, the classic symptoms of acute pulpitis helps the practitioner figure out this diagnosis, which include sharp thermal sensitivity, increased pain in reclined position on account of pulpal hyperaemia and relief from pain on bathing the tooth in cold water. The patient of acute pulpitis usually comes holding a bottle of cold water. Such condition if found in any tooth, should be managed endodontically.

\subsubsection{Acute apical periodontitis}

It is a possible cause when pain may or may not be accompanied by intraoral with or without extra oral swelling. Such teeth require endodontic therapy, although if the tooth is associated with swelling, it is better to let the swelling subside before starting any further treatment. Tooth discoloration, inflamed gingiva, dull sound and tenderness on percussion as well as pain on palpation in relation to this tooth are diagnostic of this condition. The tooth may show wide caries, large restoration, attrition, cervical abrasion and gingival recession. Occult caries, though very uncommon might require preparation of test cavity to reach proper diagnosis. In some instances, chronic apical periodontitis (asymptomatic) show acute exacerbation producing severe pain, with or without swelling.

\subsubsection{Neuralgic pain}

It is a rare finding which is quite hard to diagnose at this stage but must be ruled out. Usually the clue to diagnosis lies in the history. Patient sometimes uses the word "current" to explain the nature of pain.

\subsubsection{Extraction}

Any extraction performed in the previous visit may produce similar symptoms especially if the socket wound gets infected or dry socket develops.

\subsection{Treatment}

Once the diagnosis of flare up has been established, following clinical procedures can be performed depending upon the symptoms.

\subsubsection{Re-instrumentation}

After establishment of correct working length, canals should be carefully and completely debrided, copiously irrigated, dried and packed with an intracanal medicament further to be layered upon by a temporary restoration. Incomplete debridement in form of inadequate chemomechanical preparation or short working length tends to leave pulpal remnants in case of vital pulp and infective debrii in case of necrotic pulps, which then lead to development of flare up. On the other hand, over working length explicitly inflicts periapical trauma alongwith apical extrusion of microbes, intracanal irrigants and medicaments.

*Missed canal: Radiograph should be repeated at a different angle and a second clinical effort should be made to investigate for any missed canal. Maxillary first molars are most frequently found to have an extra canal with mandibular third molars, mandibular incisors and mandibular premolars exhibiting a relatively lower frequency [Garg et al. 2010].

\subsubsection{Occlusal reduction}

Development of an acute apical abscess postoperatively causes elevation of tooth in its socket resulting in tenderness on percussion and difficulty in biting. Such teeth can be partly and quickly relieved by judicious reduction (functional cusps) of occlusion. However, various approaches have been documented, where Cohen [Cohen 1980] suggested occlusal relief prior to endododontic therapy with Ingle [Ingle 1965], Weine [Weine 1976] and Grossman [Grossman 1981] of opinion that occlusion should be relieved prior to root canal treatment in teeth, which are painful to start with. Dorn et al [Dorn 1977] advocated reduction of occlusion whenever the painful symptoms appear.

\subsubsection{Incision and drainage}

Flare ups aggravated with extraoral or intraoral swelling should be managed vigilantly using two approaches:

\subsubsection{Self drainage}

Endodontically treated teeth with raised periapical pressure, which drain the instant root canal dressing is removed, are suitable for this approach. They should be left open for couple of days to allow evacuation of tissue fluid and pus.

Dentinal shavings occasionally lodged at the apical foramen might interfere with self-drainage and should be carefully removed using a sterlised hand instrument (file or reamer).

\subsubsection{Surgically induced drainage}

Teeth with negative or scant scope of self-drainage qualify for this approach, which involves making an incision in the most dependent part of swelling followed by deliberate palpatory motions encompassing dependent area in a direction towards the incision to facilitate maximum evacuation of pus. The canals should be then dressed with appropriate medicaments after thorough irrigation.

\subsubsection{Trephination}

It refers to surgical perforation of alveolar bone to facilitate venting of accumulated apical fluid. The efficiency of this method is debatable with inadequate reports of its actual application.

\subsubsection{Therapeutics}

\subsubsection{Antibiotics}

Antibiotics though used widely in endodontics are debated to have a limited use in management of pain and swelling. However, if cellulitis, fever, malaise and toxaemia manifest, systemic antibiotics must be prescribed. Popular oral combinations are amoxicillin and potassium clavulinate, and ofloxacin and ornidazole. IV / IM route is reserved for cases where oral administration is unmanageable.

\subsubsection{Analgesics}

Non steroid anti-inflammatory drugs (NSAID's) like Paracetamol, Ibuprofen and Diclofenac are usually effective in controlling mild to moderate pain whereas, severe episodes of pain unresponsive to NSAIDs should be dealt with narcotic analgesics like pentazocine and codeine.

\subsubsection{Steroids}

Intraoral and extraoral swelling is fairly benefited by use of steroids, administered in a single dose (e.g. 4-6mg of dexamethasone) [Leisinger et al. 1993]. The anti-inflammatory activity of steroids is based on their abilities to:

- Retard lysosomal release from cells by inhibiting fusion of lysosomes with their target membranes [Goldstein 1976]. 
- Inhibit the liberation of free arachidonic acid from the phospholipids of cell membrane by phospholipases thereby preventing formation of PG's and thromboxanes.

- Inhibit the formation of leukotrienes and other oxygenated derivatives.

Unfortunately, steroids also have an effect on inflammatory cells [Smith et al. 1976] and interfere with phagocytosis and protein synthesis due to which infections may become rampant and tissue repair might be delayed or impaired.

\subsubsection{Local anaesthetics}

Breaking of pain cycle is important, both psychologically and neurophysiologically [Gordon 1997] and requires sensory nerve blockade, which might be difficult to achieve with analgesics, thereby demanding employment of long acting local anesthetics such as etidocaine or [Dunsky et al. 1984] bupivacaine.

\section{Prevention}

Certain procedural and therapeutic guidelines should be followed by clinicians to reduce or eliminate the incidence of postoperative flare ups. Although their efficacy might not be definitively established, but they are surely logical:

- Multiple visit Vs single visit - multiple visits should be preferred over single visit, with an exception to vital pulp cases showing absence of periradicular lesion and preoperative pain.

- Selection of an instrumentation technique which causes minimal extrusion of infective debris, preferably crown down technique.

- Access cavity should never be left open in the inter appoinment period as it risks development of secondary periradicular infection. Exception should be made only in cases where drainage is established through this route.

- Cases showing tenderness on percussion in the first appointment should be occlusally relieved.

- The entire procedure should be performed under aseptic conditions using rubber dam, thoroughly sterlised endodontic instruments and copious amounts of irrigants.

- Access cavity should never be dried using compressed air as it may cause apical extrusion of debris. Cotton pellets can effectively serve the purpose.

- Mechanical debridement should be completed in a single appointment.

- Interappointment dressing of canals should be performed with medicaments to facilitate maximum elimination of infective microflora.

\section{Conclusion}

The pain and swelling constituting flare up forms an unexpected and entirely undesirable bump in the smooth recovery of periradicular tissues. It not only puts the patient in a physically painful position but also seeds doubt in their minds regarding professional skills of their doctor making them reconsider their actions. Therefore, it is paramount for the surgeon to reassure the patient and help them understand that flare up is an uncommon yet expected complication of root canal therapy, which is treatable, and it does not mean failure of endodontic treatment, nor affects periradicular recovery in anyway. Possessing extensive knowledge of its contributing factors, etiopathogenesis and treatment options as well as practising preventive measures help clinicians manage and prevent this tricky situation in an effective manner with compassion and skill.

\section{Acknowledgement}

I would like to thank all my patients for aspiring me to write this article.

\section{References}

[1] Cohen S (1980) Endodontic emergencies. In Pathways of the pulp 2nd ed. (Cohen S \& Burns RC Eds.), Mosby Co, St. Louis, pp. 31.

[2] Dorn SD (1977) Treatment of the endodontic emergency: a report based on questionnare. $\mathrm{J}$ Endodon 3, 94-153. http://dx.doi.org/10.1016/S0099-2399(77)80202-1.

[3] Dunsky J \& Moore P (1984) Long-acting local anesthetics. A comparison of bupivicane and etidocaine in endodontics. J Endod 10, 457461. http://dx.doi.org/10.1016/S0099-2399(84)80270-8.

[4] Fabricius L, Dahlen G, Ohman AE \& Moller AJR (1982) Predominant indigenous oral bacteria isolated from infected root canals after varied times of closure. Scandinavian journal of dental research 90 134-144. http://dx.doi.org/10.1111/j.1600-0722.1982.tb01536.x.

[5] Garg N \& Garg A (2010) internal anatomy. In Textbook of Endodontics 2nd ed. (Garg N \& Garg A Eds.), Jaypee, New Delhi, pp. 173.

[6] Glennon JP, Ng YL, Setchell DJ \& Gulabivala K (2004) Prevalence of and factors affecting postoperation pain in patients undergoing two visit root canal treatment. Int Endod $J$ 37, 29-37. http://dx.doi.org/10.1111/j.1365-2591.2004.00748.x.

[7] Goldstein IM (1976) Pharmacologic manipulation of lysosomal enzyme release from polymorphonuclear leukocytes. J Invest Dermatol 67, 622. http://dx.doi.org/10.1111/1523-1747.ep12544414.

[8] Gordon S (1997) Blockade of peripheral neuronal barrage reduces postoperative pain. Pain 70, 209-214. http://dx.doi.org/10.1016/S0304-3959(96)03315-5.

[9] Grossman LI (1981) Endodontic Practice. 10th Edn. Lea Febiger, Philadelphia.

[10]Ingle JI (1965) an atlas of pulp and periapical biology. Lea Febiger, Philadelphia.

[11]Leisinger A, Marshall FJ \& Marshall JG (1993) Effect of variable doses of dexamethasone on post treatment endodontic pain. J Endod 19, 35-39. http://dx.doi.org/10.1016/S0099-2399(06)81039-3.

[12]Levin L, Amit a \& Ashkenazi M (2006) Post-operative pain and use of analgesic agents following various dental procedures. American Journal of Dentistry 19, 245-247.

[13]Oliveira Alves V (2010) Endodontic flare - ups: a prospective study. Med Oral Patol Oral Cir Bucal 110, 68-72.

[14]Pasqualini D, Mollo L, Scotti N, Cantatore G, Castellucci a \& Migliaretti G (2012) Postoperative pain after manual and mechanical glide path: a randomized clinical trial. J Endod 38, 32-6. http://dx.doi.org/10.1016/j.joen.2011.09.017.

[15] Sathorn C (2007) the prevalence of postoperative pain and flare-up in single and multiple-visit endodontic treatment: a systematic review. Int Endod J 10, 1-9. http://dx.doi.org/10.1111/j.13652591.2007.01316.x

[16] Sathhorn C, Parashos P \& Messer H (2007) the prevalence of postoperative pain and flare-up in single and multiple-visit endodontic treatment; a systemic review. International Endodontic Journal 41, 91-99.

[17] Selye $H$ (1953) the part of inflammation in the local adaptation syndrome. In The mechanism of inflammation (Jasmin G \& Robert an Eds.), Acta Montreal, pp. 53-74.

[18]Senia ES (2000) Canal diameter: the forgotten dimension. Endod Prac 3, 34-8.

[19]Siqueira JF Jr. (2001) Aetiology of root canal treatment failure: why well-treated teeth can fail. International endodontic journal 34, 1-10. http://dx.doi.org/10.1046/j.1365-2591.2001.00396.x.

[20]Siqueira JF Jr., Uzeda M \& Fonseca MEL (1996) Scanning electron microscopic evaluation of in vitro dentinal tubules penetration by selected anaerobic bacteria. Journal of Endodontology 22, 308-10. http://dx.doi.org/10.1016/S0099-2399(96)80265-2.

[21] Smith RG, Patterson SS \& El-Kafrawy AH (1976) Histologic study of the effects of hydrocortisone on the apical peridontium of dogs. J Endodon 2, 376. http://dx.doi.org/10.1016/S0099-2399(76)80101-X.

[22]Udoye $\mathrm{CH} \&$ Aguwa E (2010) Flare - up incidence and related factors in adults. J Dent Oral Hyg 2, 19-22.

[23] Van Wijk J \& Hoogstraten J (2006) Reducing fear of pain associated with endodontic therapy. International Endodontic Journal 39, 384 388. http://dx.doi.org/10.1111/j.1365-2591.2006.01090.x.

[24]Weine FS (1976) Endodontic therapy. 2nd edn. Mosby Co, St. Louis: CV.

[25]Zuckerman O, Metzger Z, Sela G \& Lin S (2007) “Flare-up" during endodontic treatment: etiology and management. Refuat Hapeh Vehashinayim 24, 19-26. 